\title{
VARIABLE STRUCTURE CONTROL OF CONSTRAINED DYNAMIC SYSTEMS
}

\author{
Han-Pang Huang Marlon Lin \\ Robotics Laboratory \\ Department of Mechanical Engineering \\ National Taiwan University \\ Taipei, Taiwan 10764, R.O.C.
}

\begin{abstract}
A typical example of constrained dynamic systems is the constrained robot system in which the motion of the robot endeffector is restrained by environment. Such a system is usually composed of a set of differential equations and a set of algebraic equations. A modified computed torque controller has been developed by McClamroch and Wang. If the mathematical model of the robot is exact, the modified computed torque can simultaneously control the robot motion and contact force in an accurate way. However, there may exist uncertainties in the model, such as flexibility of joints and links, joint friction, and inexact surface model. It will be shown that the modified computed torque controller may result in an unstable closedloop system for the system with uncertainties. This difficulty can be overcome by using variable structure controller. The controller is robust in that it is insensitive to variations in the plant parameters and to external disturbances of contact force.
\end{abstract}

\section{Introduction}

In many cases, the robot end-effector interacts with environment. Hence, the robot motion is constrained. Such system is a typical example of constrained dynamic systems. For a constrained system, simultaneous control of position and interacting contact force is required. In order to investigate this problem, the contact force can be incorporated into the robot model to form a so-called singular system $[1,2]$. Several control strategies have been developed on the basis of this singular model $[3,10,11]$. In those approaches, the models for the robot system and environment are assumed exact; however, it is not generally true. For instance, the system may be subjected to uncertainties due to joint friction, joint and link flexibilities, surface friction, and compliant surface. Under these circumstances, the approaches described in $[3,10,11]$ may be inadequate or even result in unstable system. In order to overcome the above difficulty, a robust controller is proposed for the constrained dynamic system. The controller is variable structure type per se. It is insensitive to the variation of system parameters and external disturbance; moreover, the chattering effect can be eliminated in the controller design.

This paper is organized as follows: The constrained dynamic system and some preliminary results are first presented. Next, the variable structure controller is designed on the basis of nonsingular system model. Then the variable structure controller is designed for simultaneous control of position and contact force with the consideration of system uncertainties. Finally, an example for equality constrained system is given. Comparisons between the variable structure controller and the modified computed torque controller are also made. It will be shown that the variable structure controller is robust to unmodelled dynamics, while the computed torque controller may result in unstable systems.

\section{Problem Formulation and Preliminaries}

The robot system under consideration is described by equations of the type $[1,2,10]$

$$
\begin{gathered}
M(q) \ddot{q}+F(q, \dot{q})=u+J^{T}(q) \lambda \\
\phi(q)=0
\end{gathered}
$$

where $q \in R^{n}$ is the generalized displacement; $M(q)$ is an $n \times n$ inertial matrix function; $F(q, \dot{q})$ is an $\mathrm{n}$ dimensional vector function, containing the Coriolis, the centrifugal and the gravitational terms; $u \in R^{n}$ is the generalized control input; $\phi(q)$ is the $\mathrm{m}$ dimensional constraint vector function; $J(q)=\frac{\partial \phi(q)}{\partial q}$ is an $m \times n$ Jacobian matrix; $\lambda \in R^{m}$ is the generalized contact force vector associated with the constraints.

For a linear time-invariant differential system, the variable structure controller [4-8] is directly applicable. However, the system under consideration is nonlinear as well as differentialand-algebraic type. These characteristics make the concept of variable structure controller fail to apply. In order to facilitate the following developments, the system (1) (2) is first converted into a nonsingular model by eliminating the contact force; then the design of variable structure controller is based on this nonsingular model. The other approach is to convert the system into two subsystems; then the variable structure controller is designed for both position and force control.

The nonsingular model will be first derived. Since the constraint $\phi(q)$ is twice continuously differentiable, the first and second time derivatives of the constraint are also equal to zero; i.e.,

$$
J(q) \dot{q}=0
$$

and

$$
J(q) \ddot{q}+\dot{J}(q) \dot{q}=0
$$

where $\dot{J}(q)=\frac{d}{d t} J(q)$. From Eqns. (1) and (4), the contact force can be obtained as

$$
\lambda=\left[J M^{-1} J^{T}\right]^{-1} \cdot\left[J M^{-1} F-J M^{-1} u-j_{\dot{q}}\right]
$$

Here we assume that $\left[J M^{-1} J^{T}\right]$ is nonsingular. Note that the argument in equations are omitted hereafter for simplicity. Define

$$
\begin{aligned}
& K(q)=I_{n}-J^{T}\left(J M^{-1} J^{T}\right)^{-1} J M^{-1} \\
& H(q, \dot{q})=K F+J^{T}\left(J M^{-1} J^{T}\right)^{-1} \dot{j} \dot{q}
\end{aligned}
$$




$$
R=K u
$$

Then the singular system $(1)(2)$ is converted into the following nonsingular system

$$
M(q) \ddot{q}+H(q, \dot{q})=R
$$

The first controller will be developed in terms of this nonsingular model.

Next, a transformed model for the system can be derived by using a nonlinear transformation [3] as

$$
\begin{aligned}
E_{1} \bar{M}\left(x_{2}\right) E_{2}^{T} \ddot{x}_{2}+E_{1} \bar{F}\left(x_{2}, \dot{x}_{2}\right)= & E_{1} T^{T}\left(x_{2}\right) u \\
& +E_{1} T^{T}\left(x_{2}\right) J^{T}\left(x_{2}\right) \lambda \\
E_{2} \bar{M}\left(x_{2}\right) E_{2}^{T} \ddot{x}_{2}+E_{2} \bar{F}\left(x_{2}, \dot{x}_{2}\right) & =E_{2} T^{T}\left(x_{2}\right) u \\
x_{1} & =0
\end{aligned}
$$

where

$$
\begin{gathered}
\bar{M}\left(x_{2}\right)=T^{T}\left(x_{2}\right) M\left(Q\left(x_{2}\right)\right) T\left(x_{2}\right) \\
\bar{F}\left(x_{2}, \dot{x}_{2}\right)= \\
T^{T}\left(x_{2}\right)\left[F\left(Q\left(x_{2}\right), T\left(x_{2}\right) \dot{x}_{2}\right)\right. \\
\left.+M\left(Q\left(x_{2}\right)\right) \dot{T}\left(x_{2}\right) \dot{x}_{2}\right]
\end{gathered}
$$

Our problem turns out to design the variable structure controller for the transformed system (8)-(10).

III. Design of Variable Structure Controller

The theory of variable structure system was proposed by Itkis [4]. It utilizes a so-called sliding surface to integrate two unstable systems into a stable system. When the representative point slides to origin along the sliding surface, i.e., the system enters the sliding mode, the trajectory is kept on the sliding surface on account of continuous switch of the gain between positive and negative values. In the mean time, the system response follows the motion of sliding mode instead of original system equations. Therefore, the variable structure system is robust to the variation of system parameters and external disturbances. The change of system dynamics in the variable structure system is quite similar to the constrained dynamic system. In order to design an adequate variable structure controller, the design parameters should be suitably chosen (must satisfy Routh-Hurwitz criterion ) to form the sliding surface; furthermore, the controller should satisfy the sliding condition

$$
\boldsymbol{s} \cdot \dot{\boldsymbol{s}} \leq-\eta|\boldsymbol{s}|
$$

where $s=0$ is the sliding surface, and $\eta$ is a positive scalar. If the sliding condition is met, then the sliding mode exists and the representative point can reach the sliding surface in finite time.

Since the constrained dynamic system is a singular system in nature, the typical design of variable structure controller can not be directly applied. In order to resolve this problem, two approaches are adopted: One is to convert the constrained dynamic system into a nonsingular system, the other is to utilize the transformed subsystems.

\section{Controller Design by Using Nonsingular Model}

The nonsingular model of the system has been obtained in Eq.(7). According to Eq.(7), the design of variable structure controller can be proceeded in the following way.

Let $q_{d}(t)$ denote the desired trajectory. Assume the sliding surface is chosen as

$$
S=\left[\begin{array}{llll}
s_{1} & s_{2} & \cdots & s_{n}
\end{array}\right]^{T}=C\left(q-q_{d}\right)+\left(\dot{q}-\dot{q}_{d}\right)=0
$$

where $C=\operatorname{diag}\left[c_{1} c_{2} \cdots c_{n}\right], c_{i}>0, i=1,2, \cdots, n$.

By differentiating,

$$
\dot{S}=C\left(\dot{q}-\dot{q}_{d}\right)+\left(\ddot{q}-\ddot{q}_{d}\right)
$$

Multiply both sides of (15) by M(q) and use Eq.(7), we have

$$
M \dot{S}=M\left[C\left(\dot{q}-\dot{q}_{d}\right)-\ddot{q}_{d}\right]-H+R
$$

Suppose that $M \dot{S}$ has the form

$$
M \dot{S}=\left[\begin{array}{c}
-P_{1} \operatorname{sgn}\left(s_{1}\right) \\
\vdots \\
-P_{n} \operatorname{sgn}\left(s_{n}\right)
\end{array}\right] \triangleq-A S
$$

where

$$
\begin{gathered}
A=\operatorname{diag}\left[P_{i}(q, \dot{q}) \cdot \operatorname{sgn}\left(s_{i}\right) / s_{i}\right] \\
P_{i}(q, \dot{q})>0, \quad i=1,2, \cdots, n \\
\operatorname{sgn}(s)= \begin{cases}1, & s>0 \\
-1, & s<0 \\
0, & s=0\end{cases}
\end{gathered}
$$

Define the Lyapunov function $V$ as

$$
V=S^{T} M S
$$

Since the matrix $M$ is symmetric and positive definite, from Eq.(17) we obtain

$$
\begin{aligned}
\dot{V} & =\dot{S}^{T} M S+S^{T} \dot{M} S+S^{T} M \dot{S} \\
& =-2 S^{T}\left(A-\frac{\dot{M}}{2}\right) S
\end{aligned}
$$

The sliding surface $S=0$ will be asymptotically stable as long as $\left(A-\frac{\dot{A}}{2}\right)$ matrix is positive definite. The condition can be derived as

$$
P_{i} \cdot \operatorname{sgn}\left(s_{i}\right) / s_{i}>\sum_{j=1}^{n}\left|\dot{M}_{i j} / 2\right|
$$

Let $\Delta M$ and $\Delta H$ denote the system uncertainties due to parameter variations. Then $M=M^{0}+\Delta M, H=H^{0}+\Delta H$, where $\mathrm{M}$ and $\mathrm{H}$ are estimated values; $M^{0}$ and $H^{0}$ are nominal values. Suppose that $\Delta M_{i j}, \dot{M}_{i j}$ and $\Delta H_{i}$ have the following bounds

$$
\begin{aligned}
& \left|\Delta M_{i j}\right|<\hat{M}_{i j}, \quad\left|\dot{M}_{i j}\right|<\hat{N}_{i j} \\
& \left|\Delta H_{i}\right|<\hat{H}_{i}, \quad i, j=1,2, \cdots, n
\end{aligned}
$$

Combine Eqns.(16) (17), we have

$$
M\left[c\left(\dot{q}-\dot{q}_{d}\right)-\ddot{q}_{d}\right]-H+R=\left[\begin{array}{c}
-P_{1} \operatorname{sgn}\left(s_{1}\right) \\
\vdots \\
-P_{n} \operatorname{sgn}\left(s_{n}\right)
\end{array}\right]
$$

Choose the controller $\mathrm{R}$ as

$$
\begin{aligned}
R & =-M^{0}\left[c\left(\dot{q}-\dot{q}_{d}\right)-\ddot{q}_{d}\right]+H^{0}+R^{\prime} \\
& \triangleq-M^{0} b+H^{0}+R^{\prime}
\end{aligned}
$$

where $b=\left[c\left(\dot{q}-\dot{q}_{d}\right)-\ddot{q}_{d}\right]$. Substitute (23) into (22), we get

$$
\Delta M b-\Delta H+R^{\prime}=\left[\begin{array}{c}
-P_{1} \operatorname{sgn}\left(s_{1}\right) \\
\vdots \\
-P_{n} \operatorname{sgn}\left(s_{n}\right)
\end{array}\right]
$$


or equivalently in scalar form as

$$
\sum_{j=1}^{n} \Delta M_{i j} b_{j}-\Delta H_{i}+R_{i}^{\prime}=-P_{i} \operatorname{sgn}\left(s_{i}\right)
$$

By observing condition (20), we conclude that when $S_{i}<0$, Eq.(25) becomes

$$
\sum_{j=1}^{n} \Delta M_{i j} b_{j}-\Delta H_{i}+R_{i}^{\prime}<-\theta_{i} \sum_{j=1}^{n}\left|\dot{M}_{i j} / 2\right|
$$

when $S_{i}>0$, Eq.(25) becomes

$$
\sum_{j=1}^{n} \Delta M_{i j} b_{j}-\Delta H_{i}+R_{i}^{\prime}>-s_{i} \sum_{j=1}^{n}\left|\dot{M}_{i j} / 2\right|
$$

Therefore,

$$
\operatorname{sgn}\left(s_{i}\right)\left|\sum_{j=1}^{n} \Delta M_{i j} b_{j}-\Delta H_{i}+R_{i}^{\prime}<-\right| s_{i}\left|\sum_{j=1}^{n}\right| \dot{M}_{i j} / 2 \mid
$$

The auxiliary controller $R_{i}^{\prime}$ can be further chosen as

$$
\begin{aligned}
R_{i}^{\prime}= & -\operatorname{sgn}\left(s_{i}\right)\left[\sum_{j=1}^{n} \hat{M}_{i j}\left|b_{j}\right|+\hat{H}_{i}\right] \\
& -s_{i} \sum_{j=1}^{n} \frac{\hat{N}_{i j}}{2}
\end{aligned}
$$

Use Eq.(26) in Eq.(23), the final controller can be obtained as

$$
\begin{aligned}
R_{i} & =-\sum_{j=1}^{n} M_{i j}^{0} b_{j}+H_{i}^{0}-s_{i} \sum_{j=1}^{n} \frac{\hat{N}_{i j}}{2} \\
& -\operatorname{sgn}\left(s_{i}\right)\left[\sum_{j=1}^{n} \hat{M}_{i j}\left|b_{j}\right|+\hat{H}_{i}\right]
\end{aligned}
$$

or equivalently in the vector form as

$$
R=-M^{0} B+H^{0}-\frac{1}{2} S_{1} \hat{N} D-S_{2}\left[\hat{M} B^{+}+\hat{H}\right]
$$

where

$$
\begin{gathered}
S_{1}=\operatorname{diag}\left[s_{1}, s_{2}, \cdots, s_{n}\right] \\
S_{2}=\operatorname{diag}\left[\operatorname{sgn}\left(s_{1}\right), \operatorname{sgn}\left(s_{2}\right), \cdots, \operatorname{sgn}\left(s_{n}\right)\right] \\
B=\left[b_{1}, b_{2}, \cdots, b_{n}\right]^{T} \\
B^{+}=\left[\left|b_{1}\right|,\left|b_{2}\right|, \cdots,\left|b_{n}\right|\right]^{T} \\
\hat{H}=\left[\hat{H}_{1}, \hat{H}_{2}, \cdots, \hat{H}_{n}\right]^{T} \\
\hat{N}=\left\{\hat{N}_{i j}\right]_{n \times n}, \quad \hat{M}=\left[\hat{M}_{i j}\right]_{n \times n}
\end{gathered}
$$

The controller (28) includes the $\operatorname{sgn}(\mathrm{s})$ function. Since $\operatorname{sgn}(s)$ is discontinuous at $s=0$, chattering phenomenon may occur in the neighborhood of the sliding surface. In order to eliminate the chattering effect, the boundary layer concept $[6,7]$ can be applied; namely, $\operatorname{sgn}(s)$ is replaced by saturation function $\operatorname{sat}(s / \epsilon)$, where

$$
\operatorname{sat}(z)= \begin{cases}1, & z>1 \\ z, & -1 \leq z \leq 1 \\ -1, & z<-1\end{cases}
$$

$\epsilon>0$ denotes the configuration of boundary layers. Larger $\epsilon$ results in better elimination of chattering effect. However, too large $\epsilon$ will reduce the system accuracy. A suitable $\epsilon$ may be selected according to the following criterion.

$$
\epsilon= \begin{cases}\epsilon_{1}, & \text { if } \epsilon_{2}\left|q-q_{d}\right| \leq \epsilon_{1} \\ \epsilon_{2}\left|q-q_{d}\right|, & \text { if } \epsilon_{2}\left|q-q_{d}\right|>\epsilon_{1}\end{cases}
$$

where $\epsilon_{1}>0, C>\epsilon_{2} \geq 0$ are constants. If the controller (28) takes into account the elimination of chattering effect, the matrix $S_{2}$ should be modified as

$$
S_{2}=\operatorname{diag}\left[\operatorname{sat}\left(s_{1} / \epsilon_{1}\right), \operatorname{sat}\left(s_{2} / \epsilon_{2}\right), \cdots, \operatorname{sat}\left(s_{n} / \epsilon_{n}\right)\right]
$$

Since $\mathbf{R}$ is only a pseudo controller, the original controller $u$ can be recovered by using Eqns. (7)(28). If the matrix $K$ is nonsingular, the controller $u$ is

$$
u=K^{-1}(q) R
$$

otherwise

$$
u=K^{+}(q) R
$$

where $K^{+}(q)$ is the generalized inverse of $K(q)$. The above results can be summarized as

Theorem 1:

Consider the robot system (1)(2). Assume matrix $K(q)$ is nonsingular. Given any initial conditions $q(0), \dot{q}(0)$ satisfying $\phi(q)=0, \dot{\phi}(q)=0$, then the controller (31) will result in a stable closed-loop control; i.e., $q(t) \rightarrow q_{d}(t)$ as $t \rightarrow \infty$, and the contact force $\lambda$ is indirectly controlled.

Controller Design by Using Nonlinear Transformation

In the last section, the controller design is based on a nonsingular model of the system. The contact force $\lambda$ has been eliminated from the model; hence, the force can only be controlled through the control of $q$ and $\dot{q}$. In many cases, simultaneous control of both position and contact force is required. This objective can be achieved by using transformed subsystems, Eqns.(8)-(10).

Eqns.(8)(9) are rewritten below

$$
\begin{aligned}
E_{1} \bar{M}\left(x_{2}\right) E_{2}^{T} \ddot{x}_{2}+E_{1} \bar{F}\left(x_{2}, \dot{x}_{2}\right)= & E_{1} T^{T}\left(x_{2}\right) u \\
& +E_{1} T^{T}\left(x_{2}\right) J^{T}\left(x_{2}\right) \lambda(33) \\
E_{2} \bar{M}\left(x_{2}\right) E_{2}^{T} \ddot{x}_{2}+E_{2} \bar{F}\left(x_{2}, \dot{x}_{2}\right)= & E_{2} T^{T}\left(x_{2}\right) u
\end{aligned}
$$

The controller is selected as

$$
\begin{aligned}
T^{T}\left(x_{2}\right) u= & E_{1}^{T} G_{f} E_{1} T^{T}\left(x_{2}\right) J^{T}\left(x_{2}\right)\left(\lambda-\lambda_{d}\right) \\
& -T^{T}\left(x_{2}\right) J^{T}\left(x_{2}\right) \lambda_{d}+\bar{F}^{0}\left(x_{2}, \dot{x}_{2}\right)+\bar{M}^{0}\left(x_{2}\right) E_{2}^{T} u_{N}
\end{aligned}
$$

where $G_{f}$ is an $m \times m$, symmetric and non-negative definite matrix; $\lambda_{d}$ is the desired contact force; $\bar{F}^{0}$ and $\bar{M}^{0}$ are nominal values of $\bar{F}$ and $\bar{M}, \bar{M}-\bar{M}^{0}=\Delta \bar{M}, \bar{F}-\bar{F}^{0}=\Delta \bar{F} ; u_{N} \in R^{n-m}$ is the variable structure controller which will be determined.

Substitute the controller (35) into Eqns.(33)(34), we obtain

$$
\begin{gathered}
E_{1} \bar{M}^{0} E_{2}^{T}\left(\ddot{x}_{2}-u_{N}\right)+E_{1} \Delta \bar{M} E_{2}^{T} \ddot{x}_{2}+E_{1} \Delta \bar{F} \\
=\left(I_{m}+G_{f}\right) E_{1} T^{T} J^{T}\left(\lambda-\lambda_{d}\right)
\end{gathered}
$$

$E_{2} \bar{M}^{0} E_{2}^{T}\left(\ddot{x}_{2}-u_{N}\right)+E_{2} \Delta \bar{M} E_{2}^{T} \ddot{x}_{2}+E_{2} \Delta \bar{F}=0$

Let

$$
\Delta \bar{M} E_{2}^{T} \ddot{x}_{2}+\Delta \bar{F}=\left(\bar{M}^{0} E_{2}^{T}\right) W
$$

where $W \in R^{n-m}$. Since $\bar{M}^{0}$ is nonsingular, it has $\mathrm{n}$ independent column vectors. $\left(\bar{M}^{0} E_{2}^{T}\right)_{n \times(n-m)}$ is also a full column 
rank matrix. Thus, W can be found from Eq.(38) by least square solution [9]

$$
\begin{aligned}
W= & {\left[\left(\bar{M}^{0} E_{2}^{T}\right)^{T}\left(\bar{M}^{0} E_{2}^{T}\right)\right]^{-1}\left(\bar{M}^{0} E_{2}^{T}\right) . } \\
& {\left[\Delta \bar{M} E_{2}^{T} \ddot{x}_{2}+\Delta \bar{F}\right] }
\end{aligned}
$$

From the above equation, $W$ is function of $x_{2}, \dot{x}_{2}$ and $\ddot{x}_{2}$. If $\left|q_{i}\right|,\left|\dot{q}_{i}\right|$ and $\mid \ddot{q}_{i}$ are bounded, then $\left|x_{2 i}\right|,\left|\dot{x}_{2 i}\right|$ and $\mid \ddot{x}_{2 i}$ are bounded. Hence, $\left|W_{i}\right|<\hat{W}_{i}$ for $\mathrm{i}=1,2, \cdots,(\mathrm{n}-\mathrm{m})$. Use (38) in (36) and (37), we have

$$
\begin{gathered}
E_{1} \bar{M}^{0} E_{2}^{T}\left[\ddot{x}_{2}-u_{N}+W\right]=\left(I_{m}+G_{J}\right) E_{1} T^{T} J^{T}\left(\lambda-\lambda_{d}\right) \\
E_{2} \bar{M}^{0} E_{2}^{T}\left[\ddot{x}_{2}-u_{N}+W\right]=0
\end{gathered}
$$

Suppose that $\left(E_{2} \bar{M}^{0} E_{2}^{T}\right)$ is a nonsingular matrix, then Eq.(41) gives

$$
\ddot{x}_{2}-u_{N}+W=0
$$

In order to design the variable structure controller, the sliding surface is chosen as

$$
\begin{aligned}
S_{i} & =\left(\frac{d}{d t}+c_{i}\right)^{2} \cdot\left\{\int_{0}^{t}\left[x_{2 i}-x_{2 d i}\right] d t\right\} \\
& =\left[\dot{x}_{2 i}-\dot{x}_{2 d i}\right]+2 c_{i}\left[x_{2 i}-x_{2 d i}\right] \\
& +c_{i}^{2} \cdot \int_{0}^{t}\left[x_{2 i}-x_{2 d i}\right] d t
\end{aligned}
$$

where $c_{i}>0, \mathrm{i}=1,2, \cdots,(\mathrm{n}-\mathrm{m})$. The integral control in the Eq.(43) is used to reduce the steady state error due to friction and disturbance. Differentiate (43) and use (42), we obtain

$$
\begin{aligned}
\dot{S}_{i} & =-W_{i}+u_{N i}-\ddot{x}_{2 d i}+2 c_{i}\left[\dot{x}_{2 i}-\dot{x}_{2 d i}\right] \\
& +c_{i}^{2}\left[x_{2 i}-x_{2 d i}\right]
\end{aligned}
$$

By observing this equation, the controller can be selected as

$$
\begin{aligned}
u_{N i} & =\ddot{x}_{2 d i}-2 c_{i}\left[\dot{x}_{2 i}-\dot{x}_{2 d i}\right] \\
& -c_{i}^{2}\left[x_{2 i}-x_{2 d i}\right]-\hat{W}_{i} \cdot \operatorname{sgn}\left(s_{i}\right)
\end{aligned}
$$

Combine Eqns.(43) (45), the following result is obtained

$$
S_{i} \cdot \dot{S}_{i}=\left[-W_{i}-\hat{W}_{i} \operatorname{sgn}\left(s_{i}\right)\right] \cdot s_{i}
$$

Since $\left|W_{i}\right|<\hat{W}_{i}$, Eq.(46) is always less than zero. The condition for sliding mode existence is satisfied. Namely, the trajectory will reach the sliding surface for any initial conditions. In addition, the chattering effect can be eliminated by replacing $\operatorname{sgn}\left(s_{i}\right)$ with $\operatorname{sat}\left(s_{i} / \epsilon_{i}\right)$. Then the controller $u_{N}$ can be denoted as

$$
u_{N}=\ddot{x}_{2 d}-2 C_{1}\left(\dot{x}_{2}-\dot{x}_{2 d}\right)-C_{2}\left(x_{2}-x_{2 d}\right)-S_{3} \cdot \hat{W}
$$

where $C_{1}=\operatorname{diag}\left[c_{1}, c_{2}, \cdots, c_{n-m}\right], C_{2}=\operatorname{diag}\left[c_{1}^{2}, c_{2}^{2}, \cdots, c_{n-m}^{2}\right]$ and $S_{3}=\operatorname{diag}\left[\operatorname{sat}\left(s_{1} / \epsilon_{1}\right), \cdots, \operatorname{sat}\left(s_{n-m} / \epsilon_{n-m}\right]\right.$. The origina controller $u$ can be obtained by substituting $u_{N}$ into Eq.(35). This variable structure type controller $\mathrm{u}$ will guarantee $x_{2} \rightarrow$ $x_{2 d}$ and $q(t) \rightarrow q_{d}(t)$ as $t \rightarrow \infty$. From Eqns.(33)(34), $\lambda(t) \rightarrow$ $\lambda_{d}(t)$ as $t \rightarrow \infty$. The result is summarized as follows: Theorem 2:

Consider system (1)(2). For any given initial conditions $q(0), \dot{q}(0)$ satisfying $\phi(q)=0$ and $\dot{\phi}(q)=0$, the controller defined by (35) results in a stable closed-loop system. Namely, $q(t) \rightarrow q_{d}(t)$ and $\lambda(t) \rightarrow \lambda(t)$ as $t \rightarrow \infty$.

\section{Effects of-unmodelled Dynamics}

In the previous development, the system model is an ideal one. The robotic arm is assumed to be rigid; the joint friction is ignored; the friction between the robot end-effector and the constraint surface is not taken into account. It is not true for practical applications. In the following, those unmodelled dynamics will be regarded as an extra input; its influence to the controller design and the position and force control will be discussed.

Suppose the joint and link compliance, friction, external disturbance, and system uncertainty can be modeled as $F_{e q}(q, \dot{q}) . \quad F_{e q}(q, \dot{q}) \in R^{n}$ is an unknown vector function with a known bound. Then the constrained dynamic system taking into account the unmodelled dynamics becomes

$$
\begin{gathered}
M(q) \ddot{q}+F(q, \dot{q})=u+J^{T}(q) \lambda+F_{e q}(q, \dot{q}) \\
\phi(q)=0
\end{gathered}
$$

If the controller design is in terms of the transformed method, then the transformed subsystems becomes

$$
\begin{gathered}
E_{1} \bar{M}^{0} E_{2}^{T}\left[\ddot{x}_{2}-u_{N}\right]+E_{1}\left[\Delta \bar{M} E_{2}^{T} \ddot{x}_{2}+\Delta \bar{F}-F_{e q}\right] \\
=\left(I_{m}+G_{f}\right) E_{1} T^{T} J^{T}\left(\lambda-\lambda_{d}\right) \\
E_{2} \bar{M}^{0} E_{2}^{T}\left[\ddot{x}_{2}-u_{N}\right]+E_{2}\left[\Delta \bar{M} E_{2}^{T} \ddot{x}_{2}+\Delta \bar{F}-F_{e q}\right]=0 \\
\Delta \bar{M} E_{2}^{T} \ddot{x}_{2}+\Delta \bar{F}-F_{e q}=\left(\bar{M}^{0} E_{2}^{T}\right) \cdot W
\end{gathered}
$$$$
\text { Let }
$$

Then follow the same procedures as in the previous section, the controller $u_{N}$ is similar to Eq.(47) except that $\hat{W}_{i}$ becomes larger in order to compensate the uncertainty $F_{e q}$. The range of $\hat{W}_{i}$ will depend on the bound of $F_{\text {eq }}$.

Corollary 3 :

Consider the constrained robot system (1)(2). Suppose the bound of the unmodelled dynamics is given. By adjusting the magnitude of $\hat{W}_{i}$, the controller defined in Eq.(35) will guarantee $q(t) \rightarrow q_{d}(t)$ and $\lambda(t) \rightarrow \lambda_{d}(t)$ as $t \rightarrow \infty$.

\section{Example}

In this section, a second order nonlinear constrained dynamic system will be used to illustrate the design and robustness of the variable structure controller (V.S.C.). The results are also compared with the computed torque controller (C.T.C.) proposed by McClamroch and Wang. It will be shown that the computed torque controller may cause unstable closedloop system under system parameter variations. While the variable structure controller performs well for all cases.

Consider the following second order nonlinear constrained system

$$
\begin{gathered}
\alpha \ddot{q}_{1}+10 q_{1} q_{2}=u_{1}+\lambda \\
\beta \ddot{q}_{2}-80 \gamma q_{2}^{2} \dot{q}_{2}=u_{2}-2 q_{2} \lambda
\end{gathered}
$$

The constraint equation is defined by

$$
\phi\left(q_{1}, q_{2}\right)=q_{1}-q_{2}^{2}=0
$$

Note that constants $\alpha, \beta, \gamma$ are system parameters; their nominal values are $\alpha=1, \beta=1$, and $\gamma=1$.

Let $q=\left[\begin{array}{ll}q_{1} & q_{2}\end{array}\right]^{T}$ and $x=\left[\begin{array}{ll}x_{1} & x_{2}\end{array}\right]^{T}$. Then the nonlinear transformation is given by

$$
\begin{gathered}
x=x(q)=\left[\begin{array}{l}
x_{1} \\
x_{2}
\end{array}\right]=\left[\begin{array}{c}
q_{1}-q_{2}^{2} \\
q_{2}
\end{array}\right] \\
T(x)=\left[\begin{array}{cc}
1 & 2 x_{2} \\
0 & 1
\end{array}\right]
\end{gathered}
$$

Using this nonlinear transformation, the transformed subsystems are obtained as 


$$
\begin{gathered}
2 \alpha x_{2} \ddot{x}_{2}+\left[10 x_{2}^{3}+2 \alpha\left(\dot{x}_{2}\right)^{2}\right]=u_{1}+\lambda \\
\left(4 \alpha x_{2}^{2}+\beta\right) \ddot{x}_{2}+\left[20 x_{2}^{4}-80 \gamma x_{2}^{2} \dot{x}_{2}+4 \alpha x_{2}\left(\dot{x}_{2}\right)^{2}\right] \\
=2 x_{2} u_{1}+u_{2} \\
x_{1}=0
\end{gathered}
$$

Two types of controllers will be constructed in terms of the transformed subsystems: one is the computed torque controller, the other is the variable structure controller.

First, the computed torque controller (C.T.C.) developed by McClamroch and Wang can be found as

$$
\begin{aligned}
T^{T} u= & \bar{M} E_{2}^{T} \ddot{x}_{2 d}-T^{T} J^{T} \lambda_{d}+\vec{F}+E_{1}^{T} G_{f} E_{1} T^{T}\left(\lambda-\lambda_{d}\right) \\
& +\bar{M} E_{2}^{T}\left[G_{v}\left(\dot{x}_{2 d}-\dot{x}_{2}\right)+G_{d}\left(x_{2 d}-x_{2}\right)\right]
\end{aligned}
$$

Since the C.T.C. does not consider the variation of system parameters, the controller is obtained by setting $\alpha=\beta=\gamma=1$

$$
\begin{aligned}
u_{1}= & 2 x_{2}\left[\ddot{x}_{2 d}+G_{v}\left(\dot{x}_{2 d}-\dot{x}_{2}\right)+G_{d}\left(x_{2 d}-x_{2}\right)\right] \\
& +\left[G_{f}\left(\lambda-\lambda_{d}\right)-\lambda_{d}\right]+\left[10 x_{2}^{3}+2\left(\dot{x}_{2}^{2}\right)\right] \\
u_{2}= & {\left[\ddot{x}_{2 d}+G_{v}\left(\dot{x}_{2 d}-\dot{x}_{2}\right)+G_{d}\left(x_{2 d}-x_{2}\right)\right] } \\
& -2 x_{2}\left[G_{f}\left(\lambda-\lambda_{d}\right)-\lambda_{d}\right]-80 x_{2}^{2} \dot{x}_{2}
\end{aligned}
$$

Thus, the closed-loop system is given by

$$
\begin{gathered}
2 \alpha x_{2} \ddot{x}_{2}+2(\alpha-1) \dot{x}_{2}^{2}-2 x_{2}\left[\ddot{x}_{2 d}+G_{v}\left(\dot{x}_{2 d}-\dot{x}_{2}\right)\right. \\
\left.+G_{d}\left(x_{2 d}-x_{2}\right)\right]=\left(1+G_{f}\right)\left(\lambda-\lambda_{d}\right) \\
\left(4 \alpha x_{2}^{2}+\beta\right) \ddot{x}_{2}-\left(4 x_{2}^{2}+1\right)\left[\ddot{x}_{2 d}+G_{v}\left(\dot{x}_{2 d}-\dot{x}_{2}\right)\right. \\
\left.+G_{d}\left(x_{2 d}-x_{2}\right)\right]+4(\alpha-1) x_{2} \dot{x}_{2}^{2}-80(\gamma-1) x_{2}^{2} \dot{x}_{2}=0
\end{gathered}
$$

Next, the variable structure controller (V.S.C.) will be constructed. The sliding surface is chosen as

$$
S=\left(\dot{x}_{3}-\dot{x}_{2 d}\right)+c\left(x_{2}-x_{2 d}\right)
$$

The V.S.C. controller can be found as

$$
\begin{aligned}
u_{1}= & 2 x_{2}\left[\ddot{x}_{2 d}-c\left(\dot{x}_{2}-\dot{x}_{2 d}\right)-\hat{W} \cdot \operatorname{sat}(S / \epsilon)\right] \\
& +\left[G_{f}\left(\lambda-\lambda_{d}\right)-\lambda_{d}\right]+\left[10 x_{2}^{3}+2 x_{2}^{2}\right] \\
u_{2}= & {\left[\ddot{x}_{2 d}-c\left(\dot{x}_{2}-\dot{x}_{2 d}\right)-\hat{W} \cdot s a t(S / \epsilon)\right] } \\
& -2 x_{2}\left[G_{f}\left(\lambda-\lambda_{d}\right)-\lambda_{d}\right]-80 x_{2}^{2} x_{2}
\end{aligned}
$$

Using this controller in the original system, the closed-loop system is obtained as

$$
\begin{gathered}
2 \alpha x_{2} \ddot{x}_{2}+2(\alpha-1) \dot{x}_{2}^{2}-2 x_{2}\left[\ddot{x}_{2 d}-c\left(\dot{x}_{2}-\dot{x}_{2 d}\right)\right. \\
-\hat{W} \cdot \operatorname{sat}(S / \epsilon)]=\left(1+G_{f}\right)\left(\lambda-\lambda_{d}\right) \\
\left(4 \alpha x_{2}^{2}+\beta\right) \ddot{x}_{2}-\left(4 x_{2}^{2}+1\right)\left[\ddot{x}_{2 d}-c\left(\dot{x}_{2}-\dot{x}_{2 d}\right)-\hat{W} \cdot \operatorname{sat}(S / \epsilon)\right] \\
+4(\alpha-1) x_{2} \dot{x}_{2}^{2}-80(\gamma-1) x_{2}^{2} \dot{x}_{2}=0
\end{gathered}
$$

In this example, the desired trajectory is planned as $q_{1 d}=$ $(t / 2-1)^{2}, q_{2 d}=t / 2-1$, and the desired contact force is $\lambda_{d}=3$. The initial conditions are $q_{2}(0)=-1, g_{2}(0)=0.5$. The design parameters are selected as $G_{v}=8, G_{d}=32, G_{f}=100$, and $C=5$. The parameters of boundary layer are set as $\epsilon_{1}=0.01$, $\epsilon_{2}=1$. Furthermore, the robot motion is bounded by $\left|q_{2}\right| \leq 1$, $\left|\dot{q}_{2}\right| \leq 1,\left|\ddot{q}_{2}\right| \leq 1$; and the range of parameter variation are $0.1 \leq \alpha \leq 2,0.1 \leq \beta \leq 2,0.1 \leq \gamma \leq 2$. Then the bound of
$\hat{W}$ can be computed as $\hat{W}=16$. Consider the real parameters are larger than the nominal values; i.e. $\alpha=\beta=\gamma=2$. In this case, C.T.C. controller becomes unstable in position and force control. While V.S.C. keeps excellent performance in position control; the maximum force error is only 0.2. The results are shown in Fig.1 to Fig.5.

\section{Conclusion}

This paper presents two types of variable structure controller design for the constrained dynamic system. One is based on the nonsingular system model, the other is based on the singular model. The former can not directly control the contact force; the later allows simultaneous control of position and contact force. The variable structure controller is compared with the computed torque controller. According to the simulation results, C.T.C. controller is sensitive to the parameter variation; while V.S.C. can handle various parameter variations. Namely, V.S.C. is robust to the system unmodelled dynamics. The implication is that the constrained dynamic system and the variable structure system may possess some common features in nature.

\section{REFERENCE}

[1] Huang, H.P., "The unified Formulation of Constrained Robot Systems," Proc. of IEEE Conf. on Robotics and Automation, Philadelphia, 1988.

[2] McClamroch, N.H., H.P. Huang, "Dynamics of a Closed Chain Manipulator," Proc. of American Control Conference, Boston, 1985.

[3] McClamroch, N.H., D. Wang, "Feedback Stabilization and Tracking of Constrained Robots," Proc. of American Control Conference, 1987.

[4] Itkis, U., Control System of Variable Structure. New York: Wiley, 1976.

[5] Utkin, V.I., " Variable Structure System with Sliding Modes," IEEE Trans. on Automatic Control, Vol. AC-22, April, 1977.

[6] Slotine, J.E., S.S. Sastry, "Tracking Control of Nonlinear Systems using Sliding Surface with Application to Robot Manipulators," Int. J. of Control, Vol. 38, No.2, 1986.

[7] Slotine, J.E., "The Robust Control of Robot Manipulators," Int. J. of Robotics Research, Vol. 4, 1985.

[8] Yeung, K.S., Y.P. Chen, "A New Controller Design for Manipulators using the Theory of Variable Structure Systems," IEEE Trans. on Automatic Control, Vol. AC-33, No. 2, Feb. 1988.

[9] Noble, B., J.W. Daniel, Applied Linear Algebra. New York: Prentice-Hall, 1977.

[10] Huang, H.P., N.H. McClamroch, "Time-Optimal Control for a Contour Following Problem." IEEE J. of Robotics and Automation, Vol.4, No.2, 140-149, April 1988.

[11] Yoshikawa, T., "Dynamic Hybrid Position Force Control of Robot Manipulators-Description of Hand Constrained and Calculation of Joint Driving Force," IEEE J. of Robotics and Automation, Vol. RA-3, No.5, 1987.

[12] Young, K.D., "Controller Design for a Manipulator using Theory of Variable Structure Systems," IEEE Trans. on System, Man, and Cybernetics, Vol. SMC-8, Feb. 1978.

[13] Huang, H.P., "Simulation of Constrained Robot Systems," National Science Council Report, No. NSC76-0401-E00214, Taiwan, R.O.C., July, 1988. 


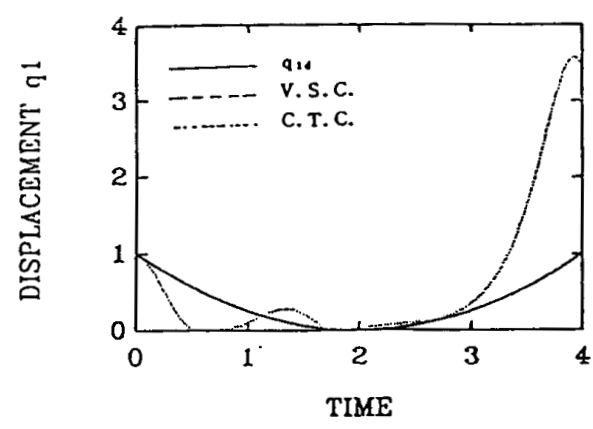

Fig.1. Displacement $q_{1}$

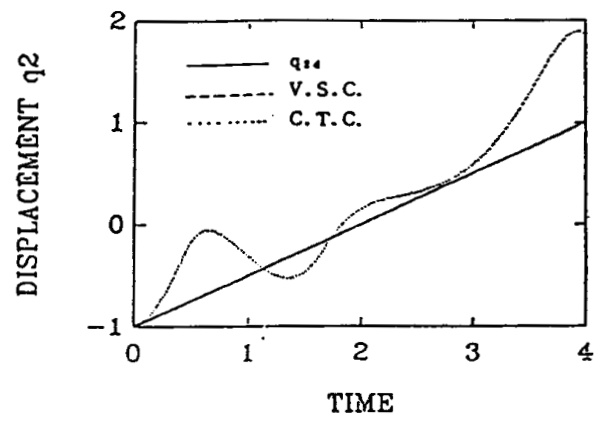

Fig.2. Displacement $q_{2}$

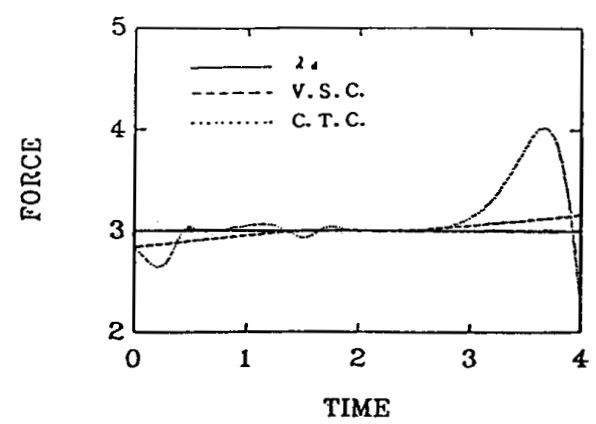

Fig.3. Contact force $\lambda$

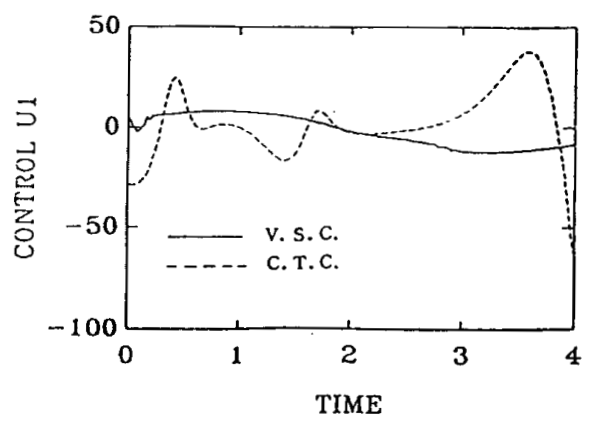

Fig.4. Input $u_{1}$

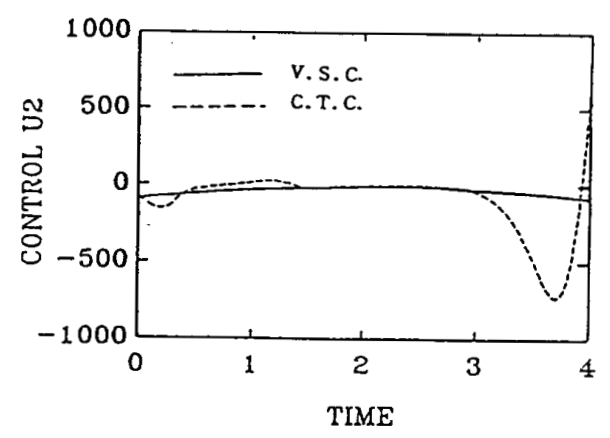

Fig.5. Input $u_{2}$ 